\title{
Prozessketten knüpfen
}

\author{
Herbert Schubert
}

In der aktuellen Organisationsentwicklung werden Areale des Siedlungsraums genutzt, um unter dem Stichwort der Sozialraumorientierung die vorhandenen professionellen Interventionen verschiedener Ressorts zu koordinieren. Im Sozialraum werden die bisher isolierten Leistungen der einzelnen Infrastrukturen über eine "Netzwerkplanung " miteinander verbunden, damit die Akteure aus Verwaltung, Bildungswesen, Gesundheitswesen, Sozialer Arbeit, Kultur etc. raum- und problemkontextbezogen wirkungsvoller und adressatenfokussiert kooperieren können.

Die Netzwerkallegorie ist gegenwärtig intensiv im Gebrauch, um den dynamischen und offenen Systemcharakter der Organisationsstrukturen in der Gesellschaft zu beschreiben. Seit den 1990er Jahren setzen sich Netzwerke als neuer Organisationsmodus durch.

Mit der Definition von Schnittstellen, der kooperativen Entwicklung gemeinsamer Produkte und Dienstleistungen und der gegenseitigen Abstimmung hilft die Netzwerkorganisation, die Defizite traditioneller Organisationsmuster des autonomen, »isolierten Handelns « zu beseitigen. Denn in der Vergangenheit wurde jedes Einzelproblem zuerst einem Funktionsbereich zugeordnet (Jugend, Soziales, Gesundheit, Bildung) und anschließend zur »Bearbeitung des Problems« ein darauf zugeschnittener Infrastrukturtyp als Betriebsform flächendeckend implementiert. Dies führte zu einer Zergliederung der institutionellen Unterstützungsstrukturen - das System der Einrichtungen und Dienstleistungen des Bildungs-, Gesundheits-, Jugend- und Sozialsektor war in der Folge fragmentiert.

Die »Netzwerkkooperation« soll einen Brückenschlag zwischen diesen operativen Inseln der Ressorts herstellen. Konstitutiv sind dabei: (1) das gemeinsames Ziel für identische Adressaten, (2) die rechtliche und wirtschaftliche Selbstständigkeit der beteiligten Akteure, (3) eine dezentrale Verantwortung der Leistungsbeiträge und (4) die Institutionalisierung des Netzwerks durch kontinuierliche Kommunikation. Auf diesem Weg kann die einzelne Organisation ihre Selbstständigkeit behalten und trotzdem Ergebnisse realisieren, zu deren Erreichen sie allein nicht in der Lage ist.

In den Feldern der Sozialwirtschaft ist Vernetzung kein neues Thema; im Laufe der vergangenen Jahrzehnte wurde immer wieder die Kooperation zwischen öffentlichen Einrichtungen und freien Trägern auf der einen Seite und Fachbereichen auf der anderen Seite angeregt. Die Vernetzungsidee war ein kontinuierlicher Treiber der Professio-

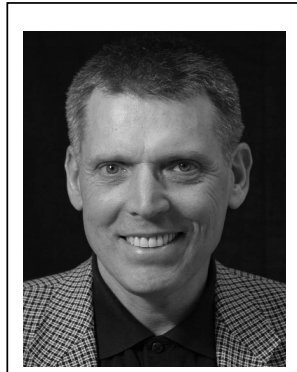

Prof. Dr. Herbert Schubert leitet in der Fachhochschule Köln das Institut für angewandtes Management und Organisation in der Sozialen Arbeit und den Forschungsschwerpunkt »Sozial । Raum I Management «. Im Blickpunkt der Forschung stehen die Fragen, wie durch Programmierungs-, Gestaltungs- und Organisationsmaßnahmen in den Sozialräumen der Städte und Gemeinden der soziale Zusammenhalt erhöht, die Potenziale in der Bevölkerung geweckt sowie aktiviert und die Kooperationsnetze der lokalen Akteure und Einrichtungen wirkungsvoll verbessert werden können. Die Ergebnisse des Forschungsschwerpunktes werden genutzt, um verantwortliche und beteiligte Akteure bei organisatorischen Entwicklungs- und Kooperationsaufgaben der sozialräumlichen Arbeit strategisch und operativ zu beraten. Internet http://www.sozial-raum-management.de

nalisierung in der Sozialen Arbeit. Die Qualitätsentwicklung von Diensten und Einrichtungen der kommunalen Daseinsvorsorge in den Sozialräumen folgt heute der Logik der Netzwerkkooperation, für die drei Aspekte prägend sind:

(a) Das Handlungssystem der Sozialwirtschaft wird in Raumeinheiten neu organisiert; diese sogenannte Sozialraumorganisation stellt ein »Reengineering « der Kommunalverwaltung aus der Anforderungsperspektive der Bürgerin und des Bürgers als Adressaten dar.

(b) Die Kooperation der Akteure im Sozialraum wird auf definierte Qualitätsstandards der Dienstleistungen ausgerichtet.

(c) In der Vernetzung von Dienstleistungen verschiedener Fachbereiche sowie von Akteuren in unterschiedlicher Trägerschaft sollen die unter dem Postulat der Adressatenund Qualitätsorientierung notwendigen Kompetenzen strategisch und operativ gebündelt werden.

Die Kopplung zuvor isolierter Organisationseinheiten zu Netzen versetzt die Beteiligten in die Lage, variabel auf den Anstieg der Geschwindigkeit der Erneuerungszyklen des Wissens und Handelns zu reagieren. Qualitätsvoller bearbeitet werden auch die Schnittstellen der professio- 
nellen Akteure, wenn die funktional getrennten Spezialisten der Fachbereiche bei der Anwendung des Wissens ihre gegenseitigen Abhängigkeiten berücksichtigen.

In der Sozialwirtschaft ist die Netzwerkorganisation insbesondere für die sozialräumliche Arbeit von hohem Wert, da Kompetenzen und Wissen der Ressorts, die an Prozessen zur Unterstützung der Adressaten beteiligt sind, an verschiedenen Orten in Netzwerken zielgesteuert und flexibel verbunden werden können.

\section{Prozessorientierte Vernetzung}

In der Netzwerkkooperation werden die zuvor unverbundenen Aktivitäten systematisch zu einer Prozesskette aneinandergereiht. In der Analyse der Anschlussfähigkeit der einzelnen Prozesse sind die Schwachstellen, unklaren Absprachen und ungeklärten Verantwortungen für Übergänge zu erkennen. Prozessketten lassen sich nach primären und sekundären (unterstützenden) Aktivitäten differenzieren: Die primären Aktivitäten beinhalten die Herstellung der Dienstleistungen, ihre Distribution unter den Adressaten und den begleitenden Service. Die sekundären Aktivitäten - wie beispielsweise die Abstimmung der Angebote unter den Einrichtungen - helfen, die primären Aktivitäten zu bündeln und abzustimmen. Im Sekundärprozess werden die Inputs für die Aktivitäten des Primärprozesses vorbereitet und bereitgestellt.

Vor diesem Hintergrund müssen in der Netzwerkkooperation zwei Aktivitätsebenen trennscharf unterschieden werden: Im Zentrum steht das operativ an Primärprozessen ausgerichtete Netzwerk (Produktions- und Dienstleistungskette); es wird unterstützt von strategisch an Sekundärprozessen ausgerichteten Netzwerken (Interessen- und Handlungskoalitionen). Vorrang gebührt der Vernetzungsebene, bei der die Sicherung der Prozess- und Ergebnisqualität gegenüber den Adressatinnen und Adressaten im Vordergrund steht. Durch die professionelle Kooperation werden einzelne - bisher isoliert erbrachte - Dienstleistungen miteinander zu operativ wirkungsvolleren Primärprozessen verbunden. Dies setzt auf der sekundären Vernetzungsebene im Sozialraum Kooperationsund Informationsprozesse für den Austausch sowie die gemeinsame Verarbeitung von Informationen unter den Organisationen und Trägern voraus.

Die Vernetzungen, in denen professionelle Ressourcen zur Bildung von Koalitionen und zur Koordination von Aktivitäten gebündelt werden, werden als tertiäre Netzwerke bezeichnet. Bei der Steuerung von tertiären Netzwerken wird auf zwei Ebenen zugleich agiert: Interorganisational betreffen die Managementaufgaben die (kooperative oder kompetitive) Steuerung von Netzwerken und intraorganisational sind zugleich die beteiligten Organisationen in den Netzwerken korrespondierend zu steuern.

\section{Steuerungsmodi von Netzwerken}

Die Steuerung von Netzwerken verfolgt das Ziel, die zugrunde liegenden komplexen Austauschbeziehungen zu gestalten. Im Allgemeinen können drei Steuerungsmodi von Netzwerken differenziert werden:

(1) Netzwerkkooperation, die vom Marktmechanismus gesteuert wird, weist eine relativ hohe Unsicherheit auf, weil das Zusammenwirken der Organisationen allein auf der Basis von marktbasierten Transaktionen erfolgt.

(2) Der zweite Modus beinhaltet die fokale Steuerung, bei der das Zusammenspiel der beteiligten Organisationen von einer medialen Instanz (nicht weisungsberechtigt) koordiniert wird.

(3) Bei der Selbstorganisation handeln die beteiligten Akteure ihr Verhalten untereinander aus und leisten einen Beitrag zur »Selbstkoordination «. Im ersten und dritten Modus lässt sich die Verknüpfung der Leistungen nicht in dem Maße anordnen und kontrollieren, wie das beim Modus der fokalen Steuerung der Fall ist, weil ein höherer Aufwand für die kommunikative Herstellung des Handlungskonsenses anfällt.

Beim Zusammenwirken von öffentlichen, sozialwirtschaftlichen und zivilgesellschaftlichen Akteuren im Sozialraum koexistieren diese Steuerungsformen, wie sich an den folgenden vier Typen tertiärer Netzwerke veranschaulichen lässt:

- (Lokales) Politiknetzwerk: Bei diesem Typ handelt es sich um die Politikverflechtung zwischen staatlichen Instanzen, öffentlichen Einrichtungen und privaten Interessengruppen (Policy-Netzwerk), getragen von machtstarken »Entscheiderinnen « und »Entscheidern«, die sich um standortgebundene Ressourcen herum konfigurieren. Die Beziehungszusammenhänge sind im Allgemeinen thematisch auf Politiksektoren fokussiert (z. B. Jugendhilfe, Gesundheit), zwischen denen schwache Brückenverbindungen bestehen. Diese Netzwerke beruhen auf einer gegenseitigen Verhaltenskontrolle nach dem Modus der Selbstorganisation.

- Strategische Allianz von Dienstleistern: Hierbei handelt es sich um eine strategische Partnerschaft mit längerfristigen Beziehungen zwischen zwei oder mehreren Organisationen, die ihre Kompetenzen im Sozialraum bündeln, um strategische (Wettbewerbs-) Vorteile zu erzielen. In der Sozialwirtschaft kooperieren Organisationen in unterschiedlicher Trägerschaft über Ressortgrenzen hinweg und bilden Koalitionen unter aktuellen oder potenziellen Wettbewerbern in fokaler oder selbstorganisierter Ausrichtung. Durch die Verbindung werden individuelle Stärken komplettiert und Schwächen kompensiert.

- Kontraktnetzwerk einer Wertschöpfungspartnerschaft: Solche Netzwerke werden entlang einer Prozesskette organisiert. Es handelt sich um eine vertikale oder diagonale Form der Zusammenarbeit zwischen dem kommunalen Auftraggeber auf der strategischen Ebene (Amt oder Fachbereich) und sozialwirtschaftlichen Unternehmen oder Trägern, die - quasi als Zulieferer - auf der operativen Ebene die vertraglich vereinbarten Dienstleistungen konzertiert in einer abgestimmten Kette erbringen. Der interinstitutionelle Einbettungskontext kann von besonderen Agenturen im Sozialraum - beispielsweise Quartiermanagement oder Sozialraumkoordination - moderiert werden, um stabile Vertrauensbeziehungen herzustellen, die eine Kooperation unter Konkurrenten ermöglichen. 
- Projektnetzwerk: Im Mittelpunkt eines Projektnetzwerkes steht die zeitlich befristete Realisierung eines komplexen Vorhabens im Sozialraum. Es ist oft auf die Beziehungen der Personen begrenzt, die die beteiligten Organisationen für die Abwicklung einer konkreten Aufgabe zu einem interorganisatorischen Projektteam zusammenstellen. Wenn die Akteure bereits über eine langjährige Kooperation vertraut sind, wird im Allgemeinen auf eine fokale Steuerung verzichtet und stattdessen eine heterarchische Form gewählt.

Im Sozialraum verlangen die widersprüchlichen Netzwerklogiken einen Spagat, wenn die machtstrategischen Verhandlungen im Politiknetzwerk mit den produktstrategischen Vernetzungsaufgaben verknüpft werden. Die Akteure können dabei in die Falle einer Paradoxie zwischen Markt und Hierarchie geraten. Denn einerseits sind die Akteure eng an den hierarchisch organisierten politisch-administrativen Bereich der Stadtverwaltung gebunden. Andererseits unterliegen sie einem Zwang, die Rolle von teilautonomen Akteuren im Netzwerk einzunehmen, obwohl die Handlungsbereiche - wie beispielsweise die Jugendhilfe - kaum nach Regeln eines teilautonomen Sozialmarktes funktionieren, sondern normative Vorgaben vom Politiknetzwerk enthalten.

Um in diesem Wechselspiel von Kooperation und Wettbewerb die Orientierung behalten zu können, muss das Zusammenspiel über ein Mehrebenenmodell unterstützt werden. Dazu ist es notwendig, dass die Steuerungsebenen bei der Netzwerkkooperation komplementär zusammenwirken:

(i) Die politischen Gremien in der Kommune übernehmen die normative Verantwortung, sie konkretisieren also die Leitziele, legen die generellen Zielrichtungen programmatisch fest und sichern die dezentralen Strukturen normativ ab (Strukturqualität).

(ii) Die strategische Verantwortung liegt bei den Fachbereichen der Kommunalverwaltung. Mit den dezentralen Akteuren müssen die Ziele für die Zielfelder Ressourcen (Input), Produkte (Output), Wirkungen und Ergebnisse (Outcome) vereinbart werden. Es wird auch Verantwor- tung für die Strukturqualität übernommen, indem die kreuzfunktionale Verbindung der Ressorts und Fachbereiche hergestellt wird.

(iii) Vor Ort, also dezentral in den Sozialräumen der Adressaten, wird die operative Verantwortung getragen. Hier sind die (räumliche) Querkoordination der Akteure verschiedener Ressorts, der Aufbau zielorientierter kleiner Handlungsnetze sowie die Produkt- und Ergebnisverantwortung anzusiedeln.

\section{Handlungsrahmen für das Netzwerkmanagement}

Eine erfolgreiche Netzwerkkooperation setzt zeitliche, finanzielle und soziale Ressourcen sowie Kompetenzen der beteiligten Promotoren voraus. Ein wesentliches Erfolgskriterium ist dabei die Einbettung der Kooperation in ein zielführendes Management. Im Allgemeinen lassen sich fünf professionelle Aufgabenbereiche entlang der Phasen des Netzwerkmanagements definieren:

- In der vorbereitenden Orientierungsphase muss sich jede einzelne Organisation über sich und ihre Situation vergewissern, wofür insbesondere die SWOT-Analyse (vgl. Kasten) mit der systematischen Klärung von Stärken und Schwächen sowie der Abwägung von Chancen und Risiken - unter besonderer Berücksichtigung der Organisationen in der Umwelt als potenzielle Kooperationspartner - geeignet ist.

- In der Phase der Initiierung eines Netzwerks werden analytische Instrumente (wie die Netzwerkanalyse) zur Diagnose des aktuellen Vernetzungsstatus und identifizierende Instrumente (wie die Stakeholderanalyse) zur Ermittlung geeigneter Kooperationspartner eingesetzt.

- In der Phase der Netzwerkplanung und des Netzwerkaufbaus sind die Handlungskompetenzen besonders auf Techniken der Zielentwicklung, des Kontraktmanagements, der Organisationsplanung und des Projektmanagements fokussiert.

- In der zentralen Phase der Realisierung der Netzwerkkooperation spielen Koordination, Controlling und Evaluation sowohl auf der Ebene des Systemmanagements als auch auf der Ebene des Ressourcenmanagements

\section{Literatur}

Aderhold, Jens (2004): Form und Funktion sozialer Netzwerke in Wirtschaft und Gesellschaft. Wiesbaden: VS Verlag für Sozialwissenschaften.

Baitsch, Christof/Müller, Bernhard (Hg.) (2001): Moderation in regionalen Netzwerken. München, Mering: Hampp Verlag.

Bauer, Petra/Otto, Ulrich (Hg.) (2005): Mit Netzwerken professionell zusammenarbeiten. Bd. 2, Institutionelle Netzwerke in Steuerungs- und Kooperationsperspektive. Tübingen: dgvt-Verlag.

Castells, Manuel (2001): Die Netzwerkgesellschaft. Opladen: Leske + Budrich.

Dahme, Heinz-Jürgen/Wohlfahrt, Norbert (Hg.) (2000): Netzwerkökonomie im Wohlfahrtsstaat. Berlin: edition sigma.
Hess, Thomas (2002): Netzwerkcontrolling. Wiesbaden: Deutscher Universitäts-Verlag.

Kraege, Rüdiger (1997): Controlling strategischer Unternehmenskooperationen. München, Mering: Rainer Hampp Verlag.

Schubert, Herbert (Hg.) (2008): Netzwerkmanagement. Koordination von professionellen Vernetzungen. Wiesbaden: VS Verlag für Sozialwissenschaften.

Schubert, Herbert/Spieckermann, Holger (2004): Standards des Quartiermanagements. Köln: Verlag Sozial Raum Management.

Sydow, Jörg (2002): Strategische Netzwerke. Wiesbaden: Gabler.

Weyer, Johannes (Hg.) (2000): Soziale Netzwerke. Konzepte und Methoden der sozialwissenschaftlichen Netzwerkforschung. München: Oldenbourg. 
eine besondere Rolle. Einen hohen Stellenwert haben Kommunikationsinstrumente innerhalb und außerhalb des Netzwerks.

Die undeutlichen Grenzen und die relativ schwache Formalisierung erschweren die Steuerung der Netzwerkkooperation. Das Management von Vernetzungen muss daher besonders auf Vertrauen und Aushandlung beruhen. Dazu eignen sich Instrumente der Moderation, weil sie Vertrauen im Netzwerk generieren und die netzinterne Kommunikation offen gestalten.

Es ist offensichtlich, dass der Kooperationszusammenhang systematisch und vorausschauend reflektiert und geplant werden muss. Zur Vorbereitung einer Netzwerkkooperation müssen Netzwerkplaner die Aufgabenstellung analysieren, die Generierung der Netzwerkpartner vorbereiten, den Ablauf ihres Ineinandergreifens vorstrukturieren und das Verfahren der gemeinsamen Zielformulierungen und Zielvereinbarungen moderieren. Die professionelle planerische Vorbereitung einer Netzwerkkooperation ist ein entscheidender Meilenstein für den Erfolg.

Ein Alleinstellungsmerkmal sozialwirtschaftlichen Handelns im Rahmen der kommunalen Daseinsvorsorge besteht darin, dass die in natürlichen Netzen gebündelten sozialen Ressourcen - wie beispielsweise die Primärnetzwerke der Familie, des Freundeskreises und vertrauter Kollegencliquen sowie die Sekundärnetzwerke der Vereine auch Gegenstand der fachlich-professionellen Primärprozesse sind. Die gelungene Vernetzung von öffentlichen, sozialwirtschaftlichen und zivilgesellschaftlichen Akteuren im Sozialraum kann als ein weiterer Erfolgsfaktor betrachtet werden.

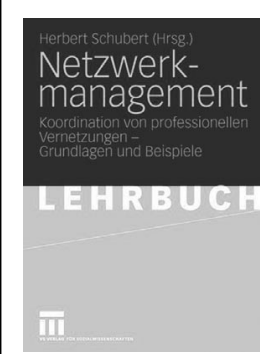

In dem Buch Netzwerkmanagement « wird das Augenmerk einerseits auf besondere Instrumente und Methoden gelegt, die für eine erfolgreiche Steuerung des netzförmigen Zusammenwirkens von Kooperationspartnern verantwortlich sind. Andererseits wird in Praxisberichten von Profit- und Non- ProfitOrganisationen veranschaulicht, wie Praktikerinnen und Praktiker die »Kunst der Steuerung« von Netzwerken handhaben. Das Spektrum reicht von Praxisnetzen im Gesundheitswesen über das Netzwerk der Offenen Ganztagsschule in Herford, das Modellprojekt »Netzwerke Früher Förderung « (NeFF) des Landschaftsverbands Rheinland, das »Mo.Ki-Netzwerk « aus Monheim und die Netzwerkstrategie der nordrhein-westfälischen Familienzentren bis hin zum Modell der Sozialraumkoordination in der Stadt Köln.

Herbert Schubert (Hg.): Netzwerkmanagement. Koordination von professionellen Vernetzungen. Grundlagen und Praxisbeispiele. VS Verlag für Sozialwissenschaften. GWV Fachverlage $\mathrm{GmbH}$, Wiesbaden 2008, ISBN 978-3-531-15444-2.

\section{Abstract}

Die Netzwerkkooperation im Sozialraum ist (a) operativ auf einen Primärprozess auszurichten (z. B. kindliche Sozialisation), (b) als Vernetzung komplementärer Kompetenzen zu entwickeln und (c) vertikal (innerhalb eines Fachbereichs) oder diagonal (über mehrere Fachbereiche hinweg) nach dem fokalen Steuerungsmodus $\mathrm{zu}$ organisieren, um das Zusammenwirken der Akteure adressaten-, qualitäts- und wirkungsbezogen zu koordinieren.

Herbert Schubert

\section{Was ist eigentlich eine SWOT-Analyse?}

Eine schnelle und anschauliche Methode Ihre persönliche und unternehmerische Ausgangslage zu klären ist die SWOT (Strenghts/Weakness/Opportunities/Threats - Stärken/Schwächen/Chancen/Risiken). Dieses Analyse-Instrument kann auch in der Gründungsphase einer selbstständigen Berufsexistenz zum Einsatz kommen.

Vorgehensweise: Die Stärken und Schwächen beziehen sich auf die gegenwärtige »interne« Situation. Bei den Chancen und Risiken betrachten Sie die »externen« Folgen, die sich daraus ergeben können. Ziel einer SWOT: Entscheidungen zu treffen, zu denen Sie stehen können. Füllen Sie, wie in dem unten stehenden Muster, die einzelnen Felder des SWOT-Analyse-Schemas aus. Ihre Eintragungen müssen nicht gleich komplett sein, sie können mit der Zeit wachsen.

\begin{tabular}{l|l}
\hline SWOT-Analyse-Schema & \\
\hline Stärken/Chancen & Schwächen/Risiken \\
\hline $\begin{array}{l}\text { Stärken/Chancen } \\
\text { Breitgefächertes und fundiertes Fachwissen im } \\
\text { Bereich XYZ }\end{array}$ & $\begin{array}{l}\text { Schwächen/Risiken } \\
\text { Keine kaufmännischen Kenntnisse }\end{array}$ \\
\hline $\begin{array}{l}\text { Chancen/Opportunities } \\
\text { Klare Experten-Positionierung möglich; gute Basis für } \\
\text { Vermarktung }\end{array}$ & $\begin{array}{l}\text { Risiken/Threats } \\
\text { Unklarheit für Honorarkalkulation; mangelnde } \\
\text { Erfolgskontrolle }\end{array}$ \\
\hline
\end{tabular}

Coaching-Tipp: Die SWOT-Analyse zeigt Ihnen immer eine momentane Einschätzung des jeweiligen Themas. Wiederholen und ergänzen Sie die SWOT in der Orientierungsphase regelmäßig, in der Gründungsphase mindestens monatlich. 